\title{
Numerical investigation to reduce environmental emissions from manufacturing industries
}

\author{
Miloua Hadj \\ Department of Mechanical engineering, Laboratory of structures Mechanics and Solids LMSS, Faculty of \\ Technology, University Djillali Liabes Sidi Bel Abbes 22000 Algeria. \\ Corresponding Author: miloua_hadj@yahoo.fr
}

Received: 30-12-2020

Accepted: 15-03-2021

\begin{abstract}
This paper aims to study carbon dioxide emissions from the cement manufacturing under wind conditions. These effects are investigated by Computational Fluid Dynamics (CFD) code used both method such as a Large Eddy Simulation (LES) and K- $\varepsilon$ methods to investigate a spread of pollution in vicinity of manufacturing. The predicted concentration, velocity and temperature of $\mathrm{CO}_{2}$ were used as the safety criterion to provide the useful information about a pollution amount resulted under variant environmental and structural conditions. A detailed look at results is beyond the scope of analysis to determine optimal $\mathrm{CO}_{2}$ emission. From the present numerical simulation the carbon dioxide appears to be the best candidate as tracer gas and Simulation (LES) methods, which directly calculate the large-scale turbulent structures and reserve modeling offer the best analysis of turbulent flows.
\end{abstract}

Key words: Manufacturing, Pollution, Atmospheric Dispersion, $\mathrm{CFD}, \mathrm{CO}_{2}$, Imposed Wind.

\section{Introduction}

We are living in a world facing serious interrelated environmental problems, including climate change, pervasive pollution, reduction in biodiversity, and looming supply constraints for a number of key natural resources. Individually, and especially in combination, these issues pose serious challenges to sustainable development. Our aim is to research on global environmental issues and emerging solutions by understanding of environmental science and related policies to increase understanding of the issues involved in tackling the environment and their ability to generate meaningful solutions. The cement industry is generally considered responsible for upwards of $5 \%$ of anthropogenic greenhouse gas emissions and is one of the leading sources of anthropogenic climate change emissions. Cement manufacturing process basically includes quarry, raw meal preparation, preheating of raw meal, kiln, clinker cooling, grinding, storage and dispatch. The basic chemistry of the cement manufacturing process begins with the decomposition of calcium carbonate $\left(\mathrm{CaCO}_{3}\right)$ at about $900^{\circ} \mathrm{C}$ to leave calcium oxide ( $\mathrm{CaO}$, lime) and liberate $\mathrm{CO}_{2}$; this process is known as calcination. This is followed by the clinkering process in which the calcium oxide reacts at high temperature (typically $1400^{\circ}-1500^{\circ} \mathrm{C}$ ) with silica, alumina and ferrous oxide to form the silicates, aluminates and ferrites respectively which forms the clinker. This clinker is then ground together with gypsum and other additives to produce cement. The largest source of carbon dioxide emissions is the material-derived $\mathrm{CO}_{2}$ driven off when the limestone is heated and decarbonised to form lime. The next largest source of $\mathrm{CO}_{2}$ emissions is the fuel used to provide the heat energy required for this chemical process (Nauclér and Enkvist, 2009). Carbon dioxide release raw materials used and large global consumption is one of the issues of international concern nowadays (Malhotra, 1999). Since The World Earth Summit 1997 in Kyoto, Japan, which initiated the need to reduce $\mathrm{CO}_{2}$ emissions on a large scale to avoid catastrophic global world, so many industrialized nations pledged to cut their yearly emissions of carbon on the way to protecting the environment (Gäbel, 2001). Several studies have been conducted by scientific community to arrive at some solutions and can aid the cement industry to achieve the higher performance in sustainable manufacturing. Sustainable manufacturing defined as the creation of manufactured products that minimize negative 
environmental impacts by implementing the concept of industrial ecology and green chemistry of cement. That are able to significantly reduce high energy consumed. The most relevant quantities used as an indicator for sustainable manufacturing evaluation in the cement industry is referred to the World Business Council for Sustainable Development (WBCSD) consisting of tones of cement per $\mathrm{MJ}$, fuel and raw material substitution rates, non product output, net $\mathrm{CO}_{2}$ per ton of cement, and incident rate. Besides, there are a number of indicators proposed by various organizations such as ISO 14031, Global Reporting Initiative (GRI), and Organization for Economic co-operation and Development (OECD).

A mathematical model to estimate the maximum reduction in carbon dioxide emissions that can be achieved by reducing energy consumption (Gäbel et al., 2004). In order to make this estimate, a minimum energy consumption of clinker production is required. Gabel et al. (2004), developed a tool to predict the environmental impact, economic cost and product performance of a facility. In a follow-up paper of Gabel et al. (2004), the researchers modeled eight scenarios in order to look for opportunities to reduce the overall environmental impact (using a life cycle analysis) number of studies have been done on the overall environmental impact of cement, including using Life Cycle Analysis techniques. Huntzinger and Eatmon (2009) considered the global warming impact to be the primary interest and Josa et al. (2007) considered that the greenhouse effect was the only global impact of cement production. Chen et al. (2010) also listed global warming impact as one of the main impacts of cement production, along with acidification, marine eco-toxicity and abiotic depletion. Both Chen et al. (2010) and Josa et al. (2007) note that the global warming impact is principally dependent on the clinker content of the cement, as it is the production of clinker that leads most of the $\mathrm{CO}_{2}$ emissions, from the decarbonized limestone and the emissions from fuel.

Some measures have been considered in order to reduce the $\mathrm{CO}_{2}$ emissions of cement industry, e.g., use of carbide slag as an alternative raw material for low carbon cement may lead to a drastic reduction. The low carbon substitutions provide significant opportunities for symbiotically utilizing large quantities of byproducts of other industrial processes reported by in paper (Reijnders, 2007). However, indirect energy use and extra emissions (including fly ash) of alternatives have not yet been fully considered during the $\mathrm{CO}_{2}$ accounting. To investigate the variation in energy consumption an energy outputs model was developed by Summerbell et al. (2016) comparing the energy output with the energy inputs as calculated from fuel consumption and its calorific value.

This work consists of a numerical study of atmospheric dispersion of carbon dioxide emissions in the cement manufacturing processes which are coming exactly from industrial stacks commonly. We have tried to reproduce the shape of a domain which represents our problem and done the numerical modeling by using the CFD software used a Large Eddy Simulation. Finally, we have tried to explain the different results which were obtained for several parameters.

\section{Mathematical models}

A flow field is characterized by the fundamental governing equations such as the continuity (Eq.1), momentum (Eq.2), energy equations (Eq.3) and species transport (Eq.4). All CFD is based on these equations:

$$
\frac{\partial \rho}{\partial \mathrm{t}}+\frac{\partial\left(\rho \mathrm{u}_{\mathrm{i}}\right)}{\partial \mathrm{x}_{\mathrm{i}}}=0
$$

$\mathrm{i}=1,2,3$ respectively for $\mathrm{x}, \mathrm{y}$ and $\mathrm{z}$

With :

$\mathrm{u}_{\mathrm{i}}$ : Components of the velocity vector $[\mathrm{m} / \mathrm{s}]$.

$\mathrm{x}_{\mathrm{i}}$ : The directions of space $[\mathrm{m}]$. 
$\rho$ : Density $\left[\mathrm{Kg} / \mathrm{m}^{3}\right]$.

$$
\frac{\partial\left(\rho u_{i}\right)}{\partial t}+\frac{\partial\left(\rho u_{i} u_{j}\right)}{\partial x_{j}}=-\frac{\partial p}{\partial x_{i}}+\frac{\partial\left(\tau_{i j}\right)}{\partial x_{j}}+\rho f_{i}
$$

With :

$\mu$ : fluid dynamic viscosity $[\mathrm{Kg} / \mathrm{m} . \mathrm{s}]$

$f_{i}$ : forces of volume $\left[N / m^{3}\right]$

p: static pressure $[\mathrm{Pa}]$

$\delta_{\mathrm{ij}}$ : Kronecker symbol $\delta_{\mathrm{ij}}=1$ si $\mathrm{i}=\mathrm{j}$ et $\delta_{\mathrm{ij}}=0$ si i $\neq \mathrm{j}$

$\tau_{\mathrm{ij}}$ : The viscous stress: $\tau_{\mathrm{ij}}=\mu\left(\frac{\partial \mathrm{u}_{\mathrm{i}}}{\partial \mathrm{x}_{\mathrm{j}}}+\frac{\partial \mathrm{u}_{\mathrm{j}}}{\partial \mathrm{x}_{\mathrm{i}}}\right)-\frac{2}{3} \mu \delta_{\mathrm{ij}} \frac{\partial \mathrm{u}_{\ell}}{\partial \mathrm{x}_{\ell}} \quad(\ell=1, \ell=2)$

$$
\rho c_{p}\left(\frac{\partial T}{\partial t}+u_{j} \frac{\partial T}{\partial x_{j}}\right)=\frac{\partial}{\partial x_{j}}\left(\lambda \frac{\partial T}{\partial x_{j}}\right)+\phi+\beta T \frac{d p}{d t}
$$

With:

T: static temperature $[\mathrm{K}]$.

$\mathrm{c}_{\mathrm{p}}$ : heat capacity of the fluid [J/Kg. K].

$\lambda$ : thermal conductivity of the fluid $[\mathrm{W} / \mathrm{m} . \mathrm{K}]$

$\beta$ : coefficient of thermal expansion $[1 / \mathrm{K}]$.

$\phi$ : Internal sources of energy dissipation $[\mathrm{W} / \mathrm{m} 2]$.

$$
\frac{\partial\left(\rho c^{\mathrm{m}}\right)}{\partial \mathrm{t}}+\frac{\partial\left(\rho \mathrm{u}_{\mathrm{j}} \mathrm{c}^{\mathrm{m}}\right)}{\partial \mathrm{x}_{\mathrm{j}}}=\frac{\partial}{\partial \mathrm{x}_{\mathrm{j}}}\left(\rho \mathrm{D}^{\mathrm{m}} \frac{\partial \mathrm{c}^{\mathrm{m}}}{\partial \mathrm{x}_{\mathrm{j}}}\right)
$$

With:

$\mathrm{D}^{\mathrm{m}}$ : molecular diffusion coefficient of the specie $\mathrm{m}\left[\mathrm{m}^{2} / \mathrm{s}\right]$.

$\mathrm{c}^{\mathrm{m}}$ : concentration of the considered species $\left[\mathrm{K} \mathrm{mol} / \mathrm{m}^{3}\right]$.

Turbulence is an extremely complex phenomenon which is still far from being modeled perfectly and on which, even with the many available studies, we have only partial results. The Reynolds number, representing the ratio between the forces of inertia and the friction exerted on a fluid, makes it possible to characterize the flow regime. For important Reynolds number and above a certain threshold, instabilities appear and the flow became disordered, the flow is then referred to as turbulent. The spatial and temporal fluctuations of different quantities turn into irregular cases with increase of the flow speed.

\subsection{Reynolds (RANS) \& Favre averaging (FANS)}

For flows with variable density, the Favre averaging is most often used for all quantities except pressure and density. To solve the system of equations a statistical approach will be used by decomposition into time-averaged and fluctuating quantities

$$
\mathrm{p}=\overline{\mathrm{p}}+\mathrm{p}^{\prime} \quad ; \quad \rho=\bar{\rho}+\rho^{\prime}
$$

While each quantity is expressed in terms of an average value (-) and a fluctuating value ('). The Favre mean For a given quantity G, is expressed as follows: 


$$
\widetilde{G}=\frac{\overline{\rho G}}{\bar{\rho}}, G=\widetilde{G}+G^{\prime \prime}
$$

So, quantities such as the speed and the concentration of a given species will be expressed as follows:

$$
\mathrm{c}^{\mathrm{m}}=\tilde{\mathrm{c}}^{\mathrm{m}}+\mathrm{c}^{\mathrm{m} "} ; \quad \mathrm{u}_{\mathrm{i}}=\tilde{\mathrm{u}}_{\mathrm{i}}+\mathrm{u}_{\mathrm{i}}{ }^{\mathrm{m}}
$$

Applying this procedure to conservation equations (Eq. 1-4):

Average Continuity Equation:

It is expressed in the following form using the equation

$$
\frac{\partial \bar{\rho}}{\partial \mathrm{t}}+\frac{\partial\left(\bar{\rho} \widetilde{\mathrm{u}}_{\mathrm{i}}\right)}{\partial \mathrm{x}_{\mathrm{i}}}=0
$$

Average Momentum Conservation Equation:

Using equation (2) we get the expression for the averaged momentum equation:

$$
\frac{\partial\left(\bar{\rho} \widetilde{\mathrm{u}}_{\mathrm{i}}\right)}{\partial \mathrm{t}}+\frac{\partial\left(\bar{\rho} \widetilde{\mathrm{u}}_{\mathrm{j}} \widetilde{\mathrm{u}}_{\mathrm{i}}\right)}{\partial \mathrm{x}_{\mathrm{j}}}=-\frac{\partial \overline{\mathrm{p}}}{\partial \mathrm{x}_{\mathrm{i}}}+\frac{\partial}{\partial \mathrm{x}_{\mathrm{j}}}\left(\bar{\tau}_{\mathrm{ij}}-\overline{\rho \mathrm{u}_{1}{ }_{1} \mathrm{u}_{\mathrm{j}}^{\prime \prime}}\right)+\left(\bar{\rho}_{\infty}-\bar{\rho}\right) \mathrm{g} \delta_{\mathrm{ij}}
$$

With :

$$
\bar{\tau}_{\mathrm{ij}}=\mu\left(\frac{\partial \widetilde{\mathrm{u}}_{\mathrm{i}}}{\partial \mathrm{x}_{\mathrm{j}}}+\frac{\partial \widetilde{\mathrm{u}}_{\mathrm{j}}}{\partial \mathrm{x}_{\mathrm{i}}}\right)-\frac{2}{3} \mu \frac{\partial \widetilde{\mathrm{u}}_{\ell}}{\partial \mathrm{x}_{\ell}} \delta_{\mathrm{ij}}
$$

Average Energy Conservation Equation:

By applying Favre's decomposition to the energy equation (3) we get the following result:

$$
\frac{\partial(\bar{\rho} \widetilde{\mathrm{T}})}{\partial \mathrm{t}}+\frac{\partial\left(\bar{\rho} \widetilde{\mathrm{u}_{\mathrm{j}}} \widetilde{\mathrm{T}}\right)}{\partial \mathrm{x}_{\mathrm{j}}}=\frac{\partial}{\partial \mathrm{x}_{\mathrm{j}}}\left(\frac{\lambda}{\mathrm{c}_{\mathrm{p}}} \frac{\partial \widetilde{\mathrm{T}}}{\partial \mathrm{x}_{\mathrm{j}}}-\overline{\rho \mathrm{u}^{\prime \prime}{ }_{\mathrm{j}}{ }^{\prime \prime}}\right)
$$

Average Species Conservation Equation:

The averaged species conservation equation using equation (4) get the following form:

$$
\frac{\partial\left(\bar{\rho} \tilde{\mathrm{c}}^{\mathrm{m}}\right)}{\partial \mathrm{t}}+\frac{\partial\left(\bar{\rho} \widetilde{\mathrm{u}}_{\mathrm{j}} \tilde{\mathrm{c}}^{\mathrm{m}}\right)}{\partial \mathrm{x}_{\mathrm{j}}}=\frac{\partial}{\partial \mathrm{x}_{\mathrm{j}}}\left(\bar{\rho} \mathrm{D}^{\mathrm{m}} \frac{\partial \tilde{\mathrm{c}}^{\mathrm{m}}}{\partial \mathrm{x}_{\mathrm{j}}}-\overline{\rho \mathrm{u}^{\prime \prime}{ }_{\mathrm{j}} \mathrm{c}^{\mathrm{m}}}\right)
$$

The averaged equations assign terms of double fluctuations correlations, they are summarized as follows:

$$
\begin{aligned}
& \overline{\rho u^{\prime \prime} u_{J}} \text { : The turbulent stress. } \\
& \overline{\rho u^{\prime \prime} T^{\prime \prime}}: \text { The thermal turbulent flow. } \\
& \overline{\rho u^{\prime \prime} c^{m n}}: \text { The mass turbulent flow. }
\end{aligned}
$$

They appear from the non-linearity of the balance equations. These terms, called Reynolds tensions, explain the effect of turbulence on the evolution of the average motion and make the systems of equations open (more unknowns than relations). It is the consequence of taking the average of the instantaneous equations which introduces a loss of information (closure of system), is the relationship between the double correlations and the average field. There are several models of closure. These models differ from each other in their order and number of equations: 
- $\quad$ The Spalart - Almaras model.

- $\quad$ The K- $\varepsilon$ model and its variants.

- $\quad$ The K- $\varepsilon$ model.

- The Reynolds stress tensor model (RSM).

- The large Eddy simulation model (LES).

- The direct numerical simulation model (DNS).

For our case we used the K- $\varepsilon$ model and the LES model.

\section{$2.2 \mathrm{k}-\varepsilon$ Model}

The k- $\varepsilon$ turbulence model still remains among the most popular, most known is the JonesLaunder $\mathrm{k}-\varepsilon$ turbulence model. To modeling the inherent term in averaging momentum equation is that the turbulent stress expressed as follows:

$$
-\overline{\rho u^{\prime}{ }_{l} u_{j}^{\prime}}=u_{t}\left(\frac{\partial u_{i}}{\partial x_{i}}+\frac{\partial u_{j}}{\partial x_{i}}\right)-\frac{2}{3}\left(\rho k+\mu_{t} \frac{\partial u_{k}}{\partial x_{k}}\right) \delta_{i j}
$$

It uses turbulent viscosity to relate Reynolds stresses and turbulent flow terms to averages flow variables, and employs the diffusion gradient hypothesis to relate Reynolds stresses to average velocity gradient and turbulent viscosity. The turbulent viscosity will therefore be expressed as a relationship between these two quantities, the turbulent kinetic energy (K) and the rate of dissipation of this energy $(\varepsilon)$. The transport equations for the model $(\mathrm{K}-\varepsilon)$ are given as follows respectively for the turbulent kinetic energy $(\mathrm{K})$ and dissipation rate $(\varepsilon)$ :

$$
\begin{gathered}
\frac{\partial}{\partial_{\mathrm{t}}}(\rho \mathrm{k})+\frac{\partial}{\partial \mathrm{x}_{\mathrm{j}}}\left(\rho \mathrm{ku}_{\mathrm{j}}\right)=\frac{\partial}{\partial \mathrm{x}_{\mathrm{j}}}\left[\left(\mu+\frac{\mu_{\mathrm{t}}}{\sigma_{\mathrm{k}}}\right) \frac{\partial \mathrm{k}}{\partial \mathrm{x}_{\mathrm{j}}}\right]+\mathrm{G}_{\mathrm{k}}+\mathrm{G}_{\mathrm{b}}-\rho \varepsilon-\mathrm{Y}_{\mathrm{M}}+\mathrm{S}_{\mathrm{k}} \\
\frac{\partial}{\partial_{\mathrm{t}}}(\rho \epsilon)+\frac{\partial}{\partial \mathrm{x}_{\mathrm{j}}}\left(\rho \varepsilon \mathrm{u}_{\mathrm{j}}\right)=\frac{\partial}{\partial \mathrm{x}_{\mathrm{j}}}\left[\left(\mu+\frac{\mu_{\mathrm{t}}}{\sigma_{\varepsilon}}\right) \frac{\partial \epsilon}{\partial \mathrm{x}_{\mathrm{j}}}\right]+\rho \mathrm{C}_{1} \mathrm{~S}_{\epsilon}-\rho \mathrm{C}_{2} \frac{\epsilon^{2}}{\mathrm{~K}+\sqrt{v \epsilon}}+\mathrm{C}_{1 \epsilon} \frac{\epsilon}{\mathrm{K}} \mathrm{C}_{3 \epsilon} \mathrm{G}_{\mathrm{b}}+\mathrm{S}_{\epsilon}
\end{gathered}
$$

With: effect).

$\mathrm{Y}_{\mathrm{M}}$ : Term taking into account the dissipation caused by the dilation (compressibility

$\mathrm{G}_{\mathrm{b}}$ : Production term due to buoyancy.

$\mathrm{G}_{\mathrm{k}}$ : Terme de production dû aux gradients moyens.

$\mathrm{S}_{\mathrm{k}}, \mathrm{S}_{\epsilon}$ : Additional source terms to be defined..

$\mathrm{C}_{3 \epsilon} \mathrm{C}_{1}, \mathrm{C}_{2}$ : Constants..

$$
\mathrm{C}_{1}=\max \left[0.43, \frac{\eta}{\eta+5}\right], \quad \eta=\mathrm{S} \frac{\mathrm{K}}{\epsilon}, \quad \mathrm{S}=\sqrt{2 \mathrm{~S}_{\mathrm{ij}} \mathrm{S}_{\mathrm{ij}}}
$$

$S$ : Tensor of the average strains 


\subsection{Large Eddy Simulation 'LES'}

(LES) which was proposed in as early as 1963 by Smagorinsky LES does not adopt the conventional time-or ensemble-averaging RANS approach with additional modeled transport equations being solved to obtain the so-called Reynolds stresses resulting from the averaging process. In LES the large scale motions $\bar{\Phi}(\mathrm{x}, \mathrm{t})$ (large eddies) of turbulent flow are computed directly and only small scale (sub-grid scale (SGS)) motions $\phi(\vec{x}, t)$ are modeled, resulting in a significant reduction in computational cost compared to DNS. LES is more accurate than the RANS approach since the large eddies contain most of the turbulent energy and are responsible for most of the momentum transfer and turbulent mixing, and LES captures these eddies in full detail directly whereas they are modeled in the RANS approach. Furthermore, the small scales tend to be more isotropic and homogeneous than the large ones, and thus modeling the SGS motions should be easier than modeling all scales within a single model as in the RANS approach. Therefore, currently LES is the most viable/promising numerical tool for simulating realistic turbulent/transitional flows (Zhiyin, 2015).

The resolved component, $\bar{\Phi}(\mathrm{x}, \mathrm{t})$, is obtained from the filtering operation by taking a function $\mathrm{G}\left(\overrightarrow{\mathrm{x}}-\overrightarrow{\mathrm{x}}^{\prime}, \Delta\right)$ as the filter kernel:

$$
\bar{\Phi}(\vec{x}, t)=\int_{\Omega} G\left(\vec{x}-\vec{x}^{\prime}, \Delta\right) \phi(\vec{x}, t) d \vec{x}^{\prime}
$$

Where $\Delta$ is the filter width related to the local mesh configuration by $\Delta=\left(\delta_{\mathrm{X}} \delta_{\mathrm{Y}} \delta_{\mathrm{Z}}\right)^{\frac{1}{3}}$ with the sizes of a grid cell $\delta \mathrm{x}, \delta \mathrm{y}$ and $\delta$ z. This means that eddies of a size larger than $\Delta$ are computed on a numerical grid, while the unresolved eddies have to be modeled.

Applying the filtering operation to each term in the conservation equations of mass, momentum, energy and species, and decomposing the dependent variables ( $u, v, w, P$,etc.) into resolved (the large-scale eddies) and subgrid components (the smallest eddies ) results in the filtered governing equations, shown below:

$$
\begin{gathered}
\frac{\partial \rho}{\partial}+\frac{\partial \rho \bar{u}_{j}}{\partial x_{j}}=0 \\
\frac{\partial \rho \bar{u}_{i}}{\partial}+\frac{\left.\partial \rho \bar{u}_{i} \bar{u}_{j}\right)}{\partial \bar{x}_{j}}+\frac{\partial \bar{P}}{\partial x_{i}}-\rho g_{i}=\nabla \cdot \bar{\tau}_{i j, S G S}
\end{gathered}
$$

Where the bar above the various quantities denotes the filtered variable. The unresolved field, $\hat{\phi}(\overrightarrow{\mathrm{x}}, \mathrm{t})$, is modeled by the Smagorinsky model (21) in which the SGS Reynolds stress tensors, $\bar{\tau}_{i j, S G S}$, are related to the local large-scale rate of strain $\bar{\tau}_{i j, S G S}=2 u_{t} \bar{S}_{i j}$ and $\bar{S}_{i j}=\frac{1}{2}\left(\frac{\partial \bar{u}_{i}}{\partial x_{j}}+\frac{\partial \bar{u}_{j}}{\partial x_{i}}\right)$. Following the analysis of Smagorinsky, the eddy viscosity can be modeled as $\mu_{t}=\rho\left(C_{S} \Delta\right)^{2}\left|\bar{S}_{i j}\right|$ Here, $\left|\bar{S}_{i j}\right|$ is the magnitude of the large-scale strain rate tensor, $\bar{S}_{i j}$, and $C_{S}$ the Smagorinsky constant, lying between 0.1 and 0.21 , is set as low as possible to reduce numerical diffusion. The turbulent heat flux is based on an eddy viscosity assumption, resulting in the following energy equation:

$$
\frac{\partial \bar{h}}{\partial}+\frac{\partial\left(\rho \bar{u}_{i} \bar{h}\right)}{\partial x_{j}}-\frac{\partial}{\partial x_{j}} \frac{\mu_{t}}{\operatorname{Pr}_{t}} \frac{\bar{h}}{\partial x_{j}}=\dot{q}_{C}^{\prime \prime \prime}-\nabla \cdot q_{r}
$$

Where $\mathbf{P r}_{t}$ denotes the turbulent Prandtl number varying from 0.2 to 0.9 , and is taken as 0.5 in this work, even though there were no significant changes by using different values in the test 
data downstream to the fire zone. The energy equation includes the rate of heat release per unit volume, $\dot{q}_{C}^{\prime \prime \prime}$, and the radiant energy flux, $\nabla \cdot q_{r}$.

\section{Results and Discussion}

In the present simulation using the CFD software to study the dispersion of flue gas from exhaust chimney in the cement manufacturing used $\mathrm{CO}_{2}$ as tracer. Two turbulence model were used to close the governed physical models which are a set of conservation equations of mass, momentum, energy, state equation, turbulent equations. Much visualization of numerical parameters were carried out at different ratios of gas ejection speed and imposed wind speed.

The geometry numerical domain taken as $\mathrm{L} \times \mathrm{W} \times \mathrm{H}=100 \mathrm{~m} \times 80 \mathrm{~m} \times 20 \mathrm{~m}$, with two industrial chimneys were simulated (see figure 1.a\& b), the first considered as an obstacle for the predominant wind and the second chimney which is the source of pollutant emission $\left(\mathrm{CO}_{2}\right)$ were used to study the atmospheric dispersion of pollutants from an industrial chimney, their heights respectively were $(\mathrm{H} 1=12 \mathrm{~m}, \mathrm{H} 2=9 \mathrm{~m})$ the buildings downstream the exhaust chimney were respectively had the dimensions $(\mathrm{L} 1 \mathrm{x} \times 11 \mathrm{y} \times \mathrm{H} 1 \mathrm{z}=10 \mathrm{~m} \times 20 \mathrm{~m} \times 15 \mathrm{~m})$ and $(\mathrm{L} 2 \mathrm{x} \times 12 \mathrm{y} \times \mathrm{H} 2 \mathrm{z}=$ $10 \mathrm{~m} \times 20 \mathrm{~m} \times 12 \mathrm{~m}$ ). The exact number Meshes is 917256 , this number were taken with Fluent code after refinement of 828376 meshes. A diagonal mesh with a refinement in disturbance or the sources zones like the emission chimney.

In this simulation, the $\mathrm{CO}_{2}$ gas was used as a tracer with $30 \mathrm{~mol} \%$ in flue gas was fixed in the cross section of discharge chimney. In practice, high $\mathrm{CO}_{2}$ concentration in cement flue gas (varied between 15 to $30 \mathrm{~mol} \%$ ) originating from a combustion of fuel in the calciner, as well as from the calcination of the raw material itself $\left(\mathrm{CaCO}_{3} \rightarrow \mathrm{CaO}+\mathrm{CO}_{2}\right)$ and from used fossil fuel. Some waste heat can be released from clinker and transported by exhaust air. In this simulation. At the start of the present simulation the air filling all domain, we considered a tracer gas $\left(\mathrm{CO}_{2}\right)$ with a concentration of $30 \mathrm{~mol} \%$ exhausted with from the chimney 1 with $9 \mathrm{~m}$ height and a vertical discharge speed at the top of the chimney fixed to $5 \mathrm{~m} . \mathrm{s}-1$ and an exhaust gas temperature equal to $400 \mathrm{~K}$, while the ambient air flow speed imposed on the inlet (Figure 1.b) varies according to the case studied (range of values) with an ambient temperature of $293.15 \mathrm{~K}$. The used turbulence models were K- $\varepsilon$ and LES. After to the appropriate selected configuration and turbulence models, we focused to the influence of wind speed on chimney stack, especially the $\mathrm{CO}_{2}$, we considered the relationship:

$\mathrm{R}=\mathrm{Vi} / \mathrm{Vv}$

with

$V_{i}=\left(\begin{array}{l}0 \\ 0 \\ 5\end{array}\right)_{(x, y, z)}$ Exhaust velocity to $5 \mathrm{~ms}-1$

$V_{v}=\left(\begin{array}{c}V x \\ 0 \\ 0\end{array}\right)_{(x, y, z)}$ wind speed varies over a margin $\mathrm{V}=\{10,5,20,3\} \mathrm{m} . \mathrm{s}-1$ 

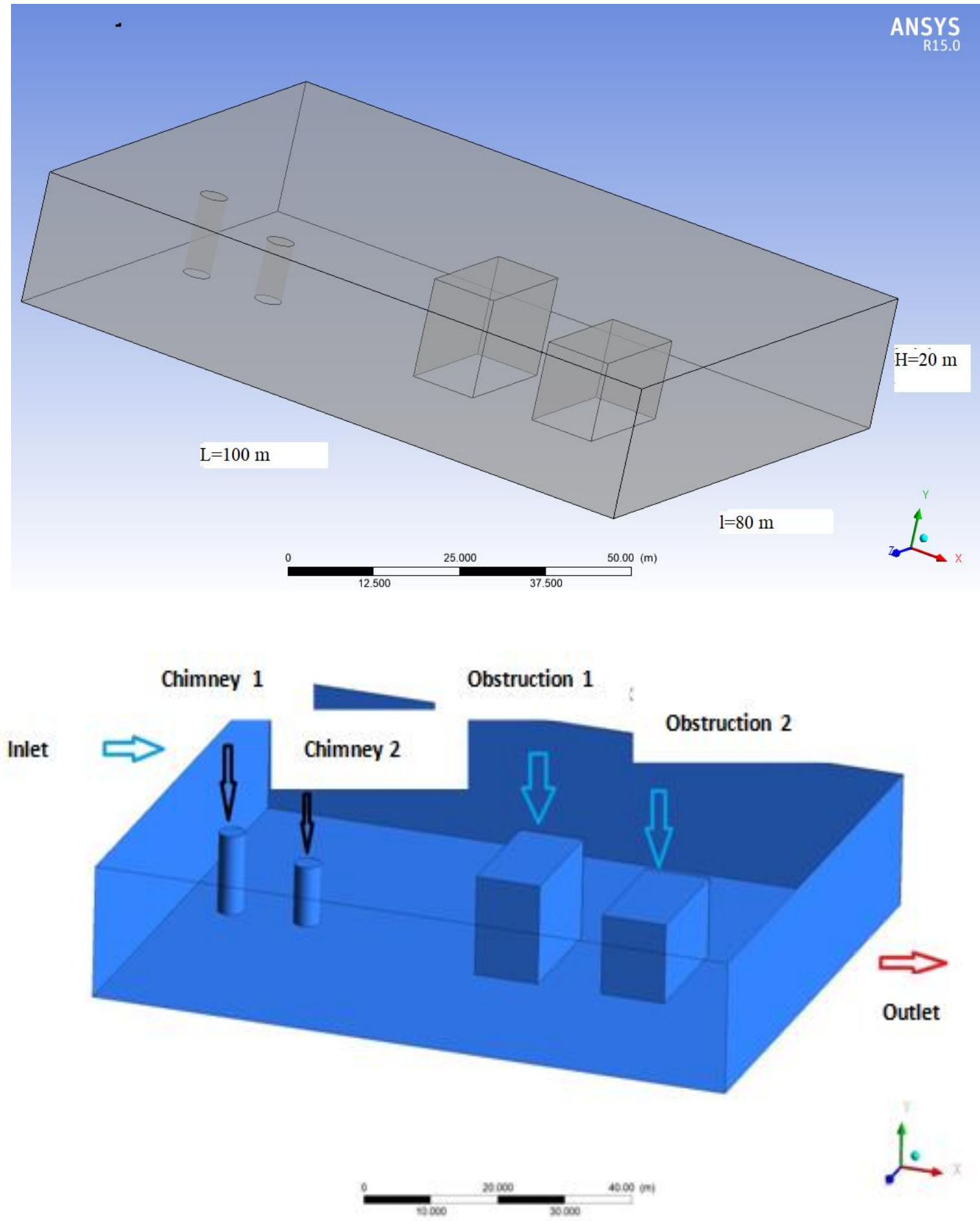

(a) 

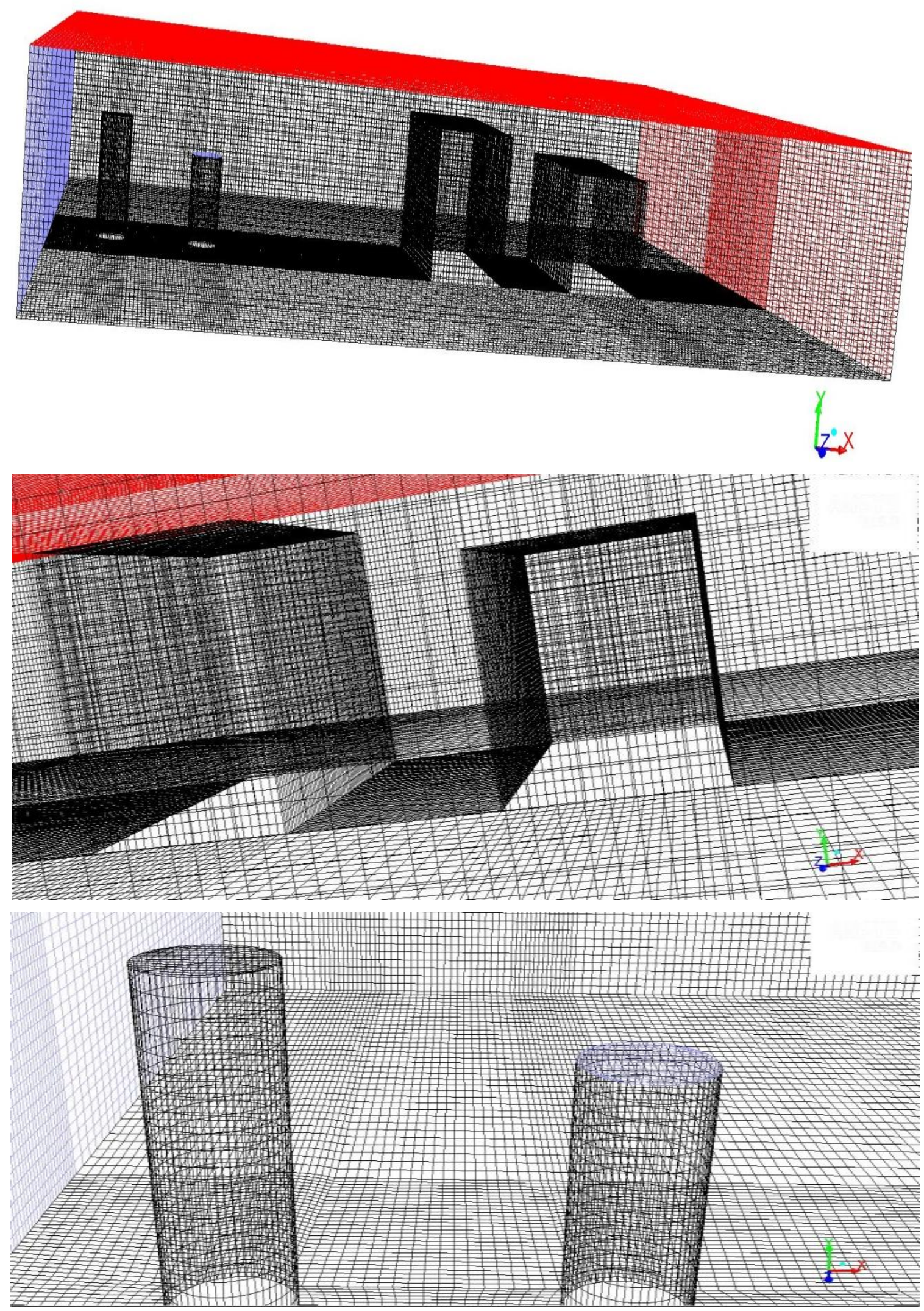

(b) 

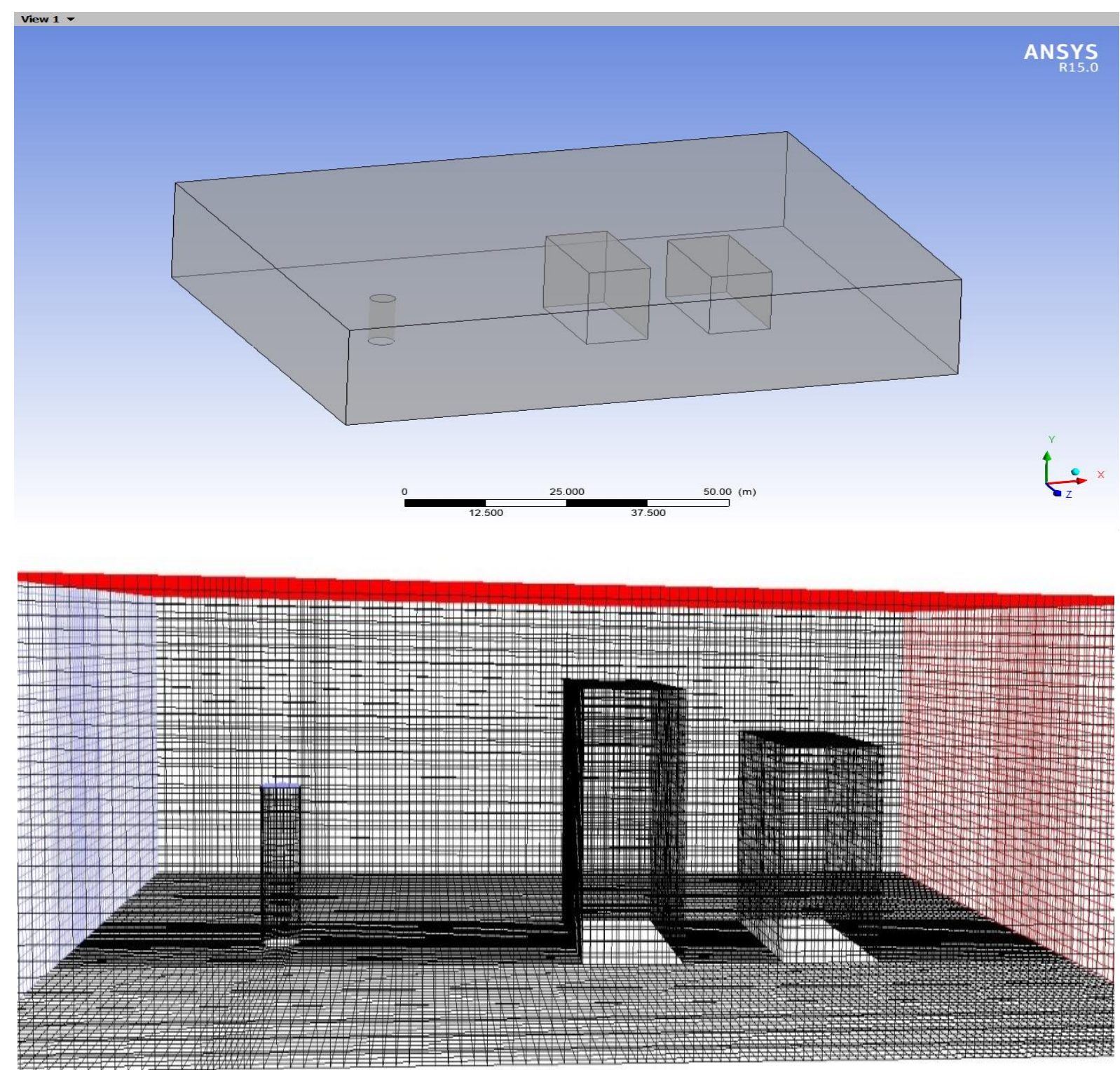

(c)

Fig. 1(a) Model geometry under CFX code, (b) Refined mesh used during the simulation with CFX (c) Geometry without chimney 1. N.B: Inlet: imposed air speed, Top: open, Exit: open, Ground: two chimneys the first plays the role of an obstacle and second is exhaust chimney' $\mathrm{CO}_{2}$ tracer' with fixer speed and two buildings downstream the exhaust chimney to study fluid-structure interaction FSI.

The simulation with K- $\varepsilon$ model (Fig.2) the wind intensity on the vertical spread of the exhaust gas was examined. The inclination and even the stretching of the pollutant plume were proportional to the increase in the wind condition with fixed exhaust velocity to $5 \mathrm{~ms}-1$. The stratified zone with Temperature $400 \mathrm{~K}$ is inversely proportional to the increase in wind speed which is justified by a primordial thermal convection which they lose its heat rapidly due to elevated GTC global transfer coefficient Transfer. Even far from to this area, the plume moves upwards due to the chimney effect. 


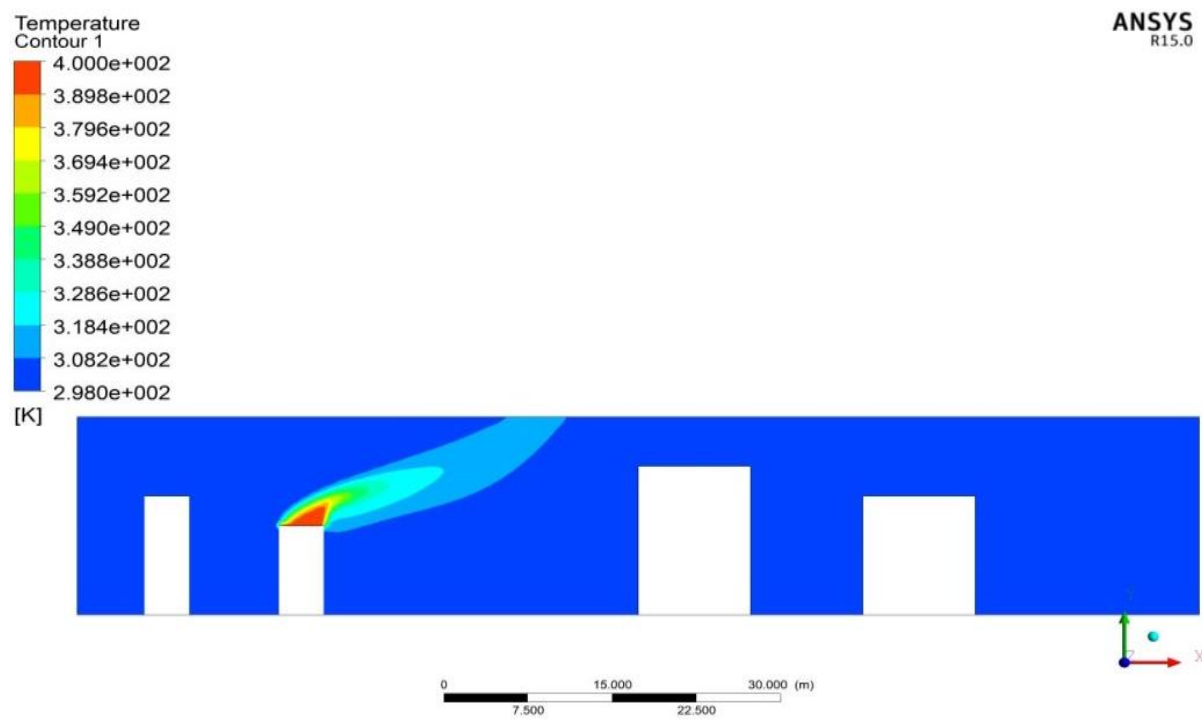

(a) $R=0.25$

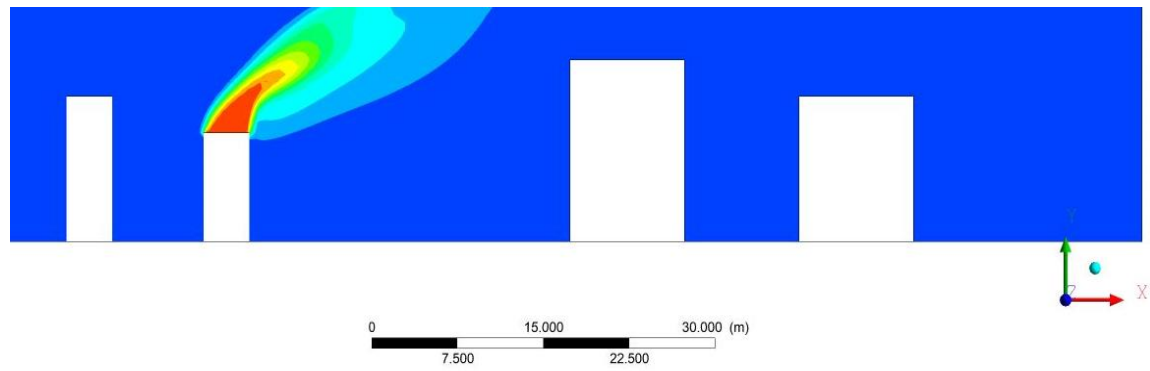

(b) $R=0.5$

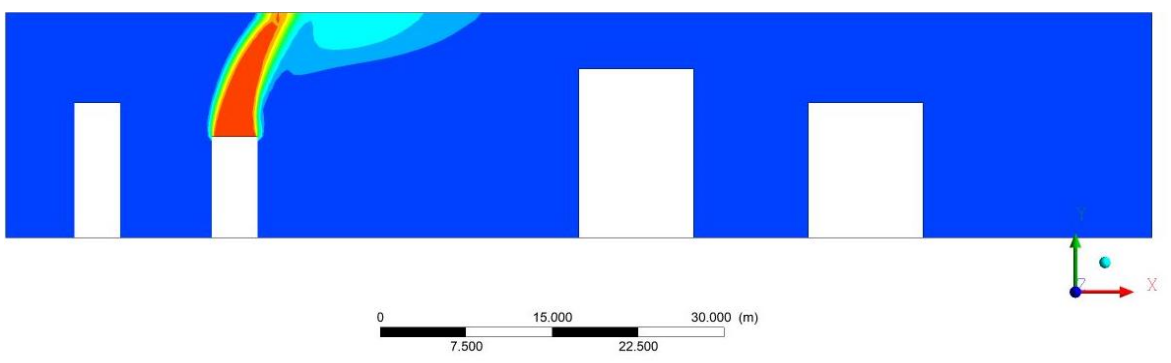

(c) $\mathrm{R}=1$

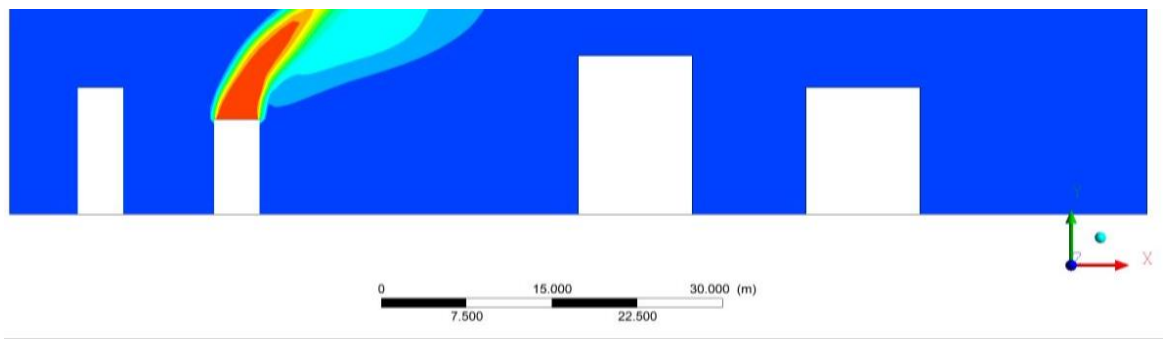

(d) $R=1.6$

Fig. 2 Influence of the speed ratio $\mathrm{R}$ on the quasistationairy shape of the plume dispersion by $\mathrm{K}-\varepsilon$ model. 
To perfectly identify the plume zones of the pollutant and its mode of dispersion, a numerical simulation using the Large Eddy Simulation model were proposed (Fig.3) to get the temporal evolution of plume dispersion. The primary advantage of this model is it possible to capture more phenomenon of dispersion even on a small scale ' modeled scale' and that the iso-contours of the variables were more clear than the K- $\varepsilon$ model which they resolve only great scale. The results indicated that the use of high velocity exhaust stacks does not guarantee adequate plume dilution and the exhaust momentum ratio, R (i.e. the ratio of exhaust velocity to wind speed), was found to significantly influence the dilution of a tracer gas emitted from a chimney with elevated wind values coming from the direction of the taller chimney.

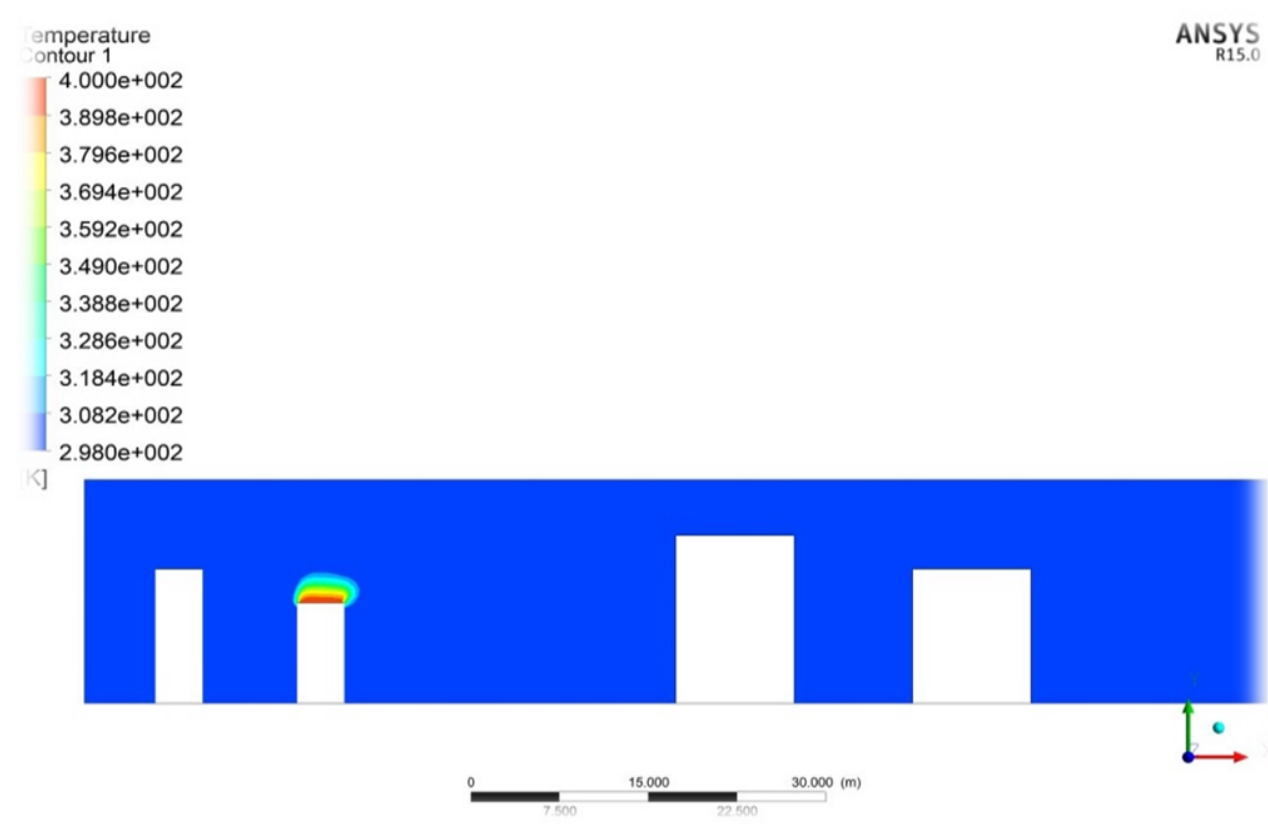

(a)

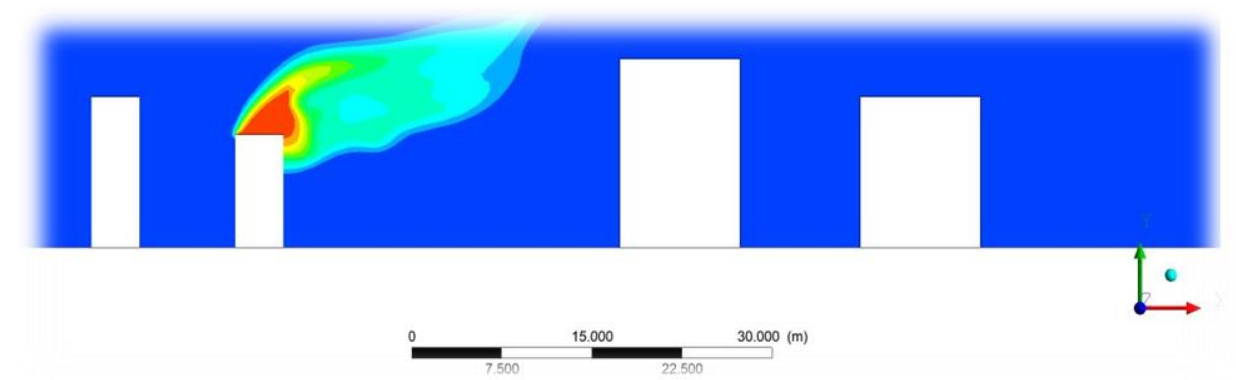

(b)

Fig. 3 Temporal dispersion of the pollutant by the LES method $\mathrm{R}=0.5$ : (a)T=0.4 , (b) $\mathrm{T}=12.7 \mathrm{~s}$.

The most significant observation is the difference in results of mean velocity between the two turbulence models even though this is the case with the wake turbulence (Fig.4). The vortex is built over time and be located near the downstream chimney 1 (obstacle) and at the top of downstream buildings, they play the role of a turbulence promoter. With LES model a more vortex become visible which they represent the black point of pollutant because it resides more than the other polluted air with linear current line. 


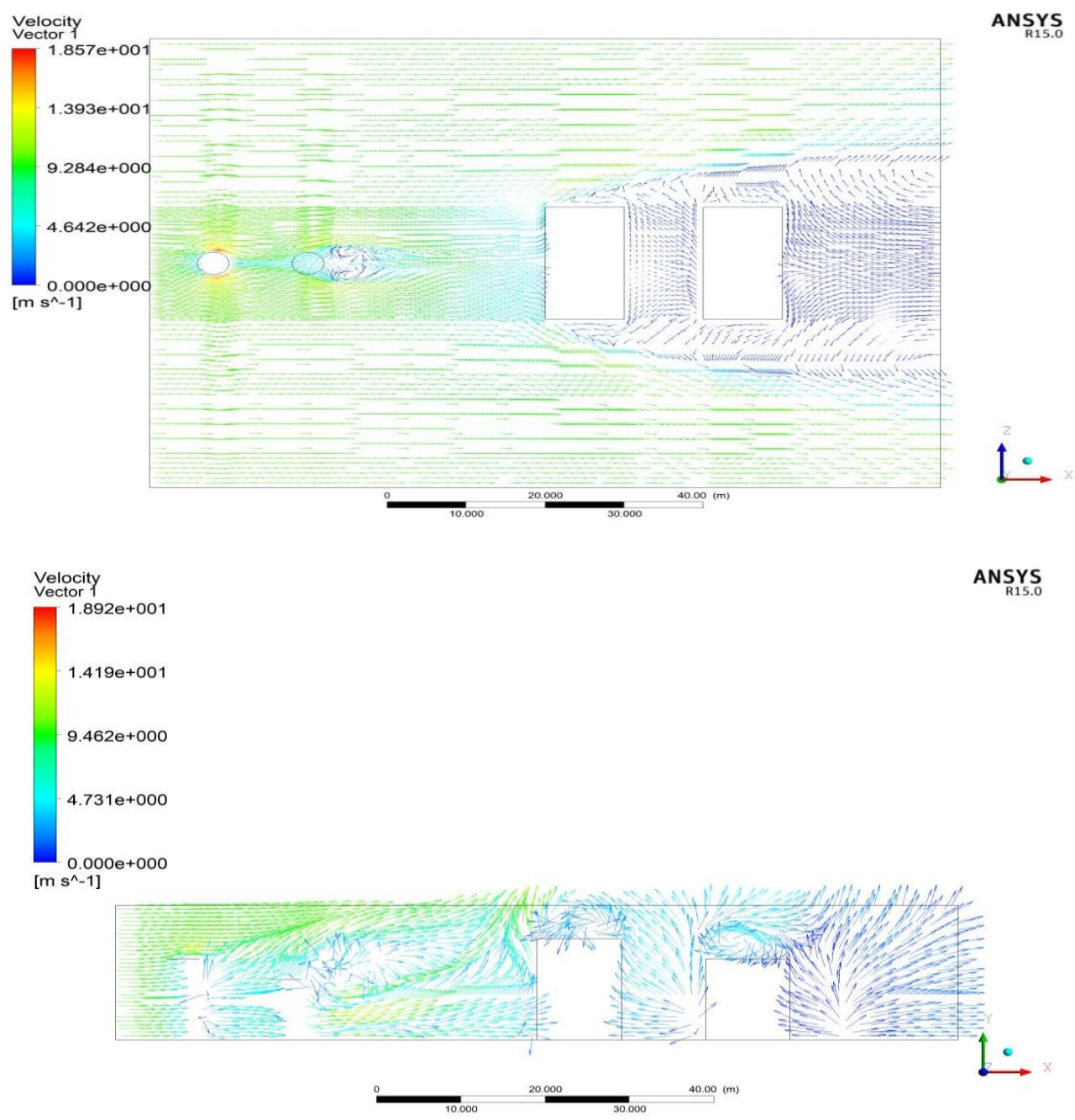

(a)

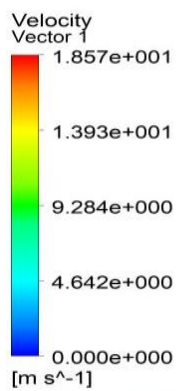

ANSYS

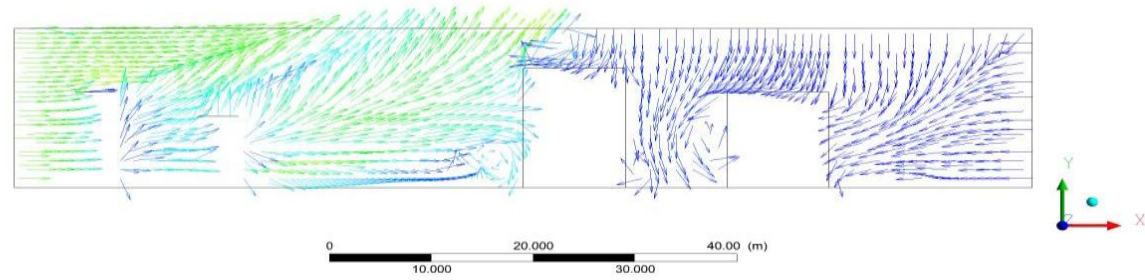

(b)

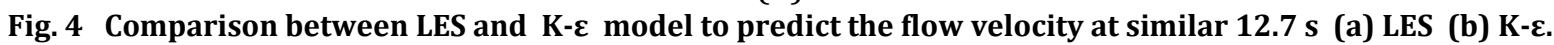

For further identification of the polluting black point i.e. the turbulent vortex we chose the XZ plane at the horizontal level to observe dispersion velocity with the test of another configuration 
of the domain without chimney $\mathrm{n}^{\circ} 1$ obstacle. For the same speed ratios $\mathrm{R}=0.5$, we established a deference velocity values and flow field between both cases without and with chimney obstacle which exists upstream exhausted chimney 2 . A deference which due to the model themselves (see Figure $5 \mathrm{a}$ and $\mathrm{b}$ ) thus the chimney $\mathrm{n}^{\circ} 1$ play the role of turbulence promoter which disturb the flow, therefore with LES model they better capture the state of flow. In order to avoid vorticity it is necessary to eliminate the chimney $n^{\circ} 1$ (see Figure. 5.c) without a vortex. So, in the construction of industrial site it is necessary from the first to avoid the random location of chimneys or buildings and to take into consideration the effect of the wind condition and their interaction with the buildings whatever their size and shape.

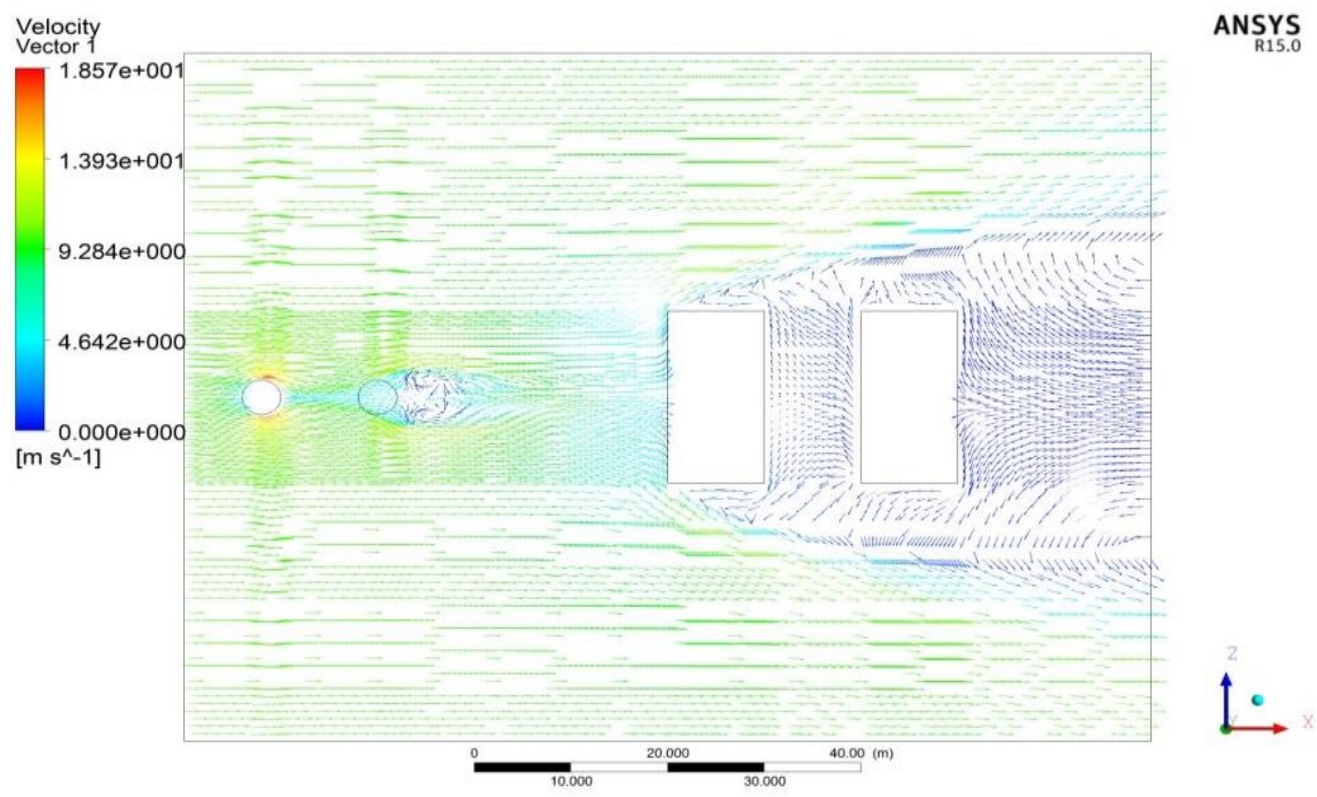

(a)

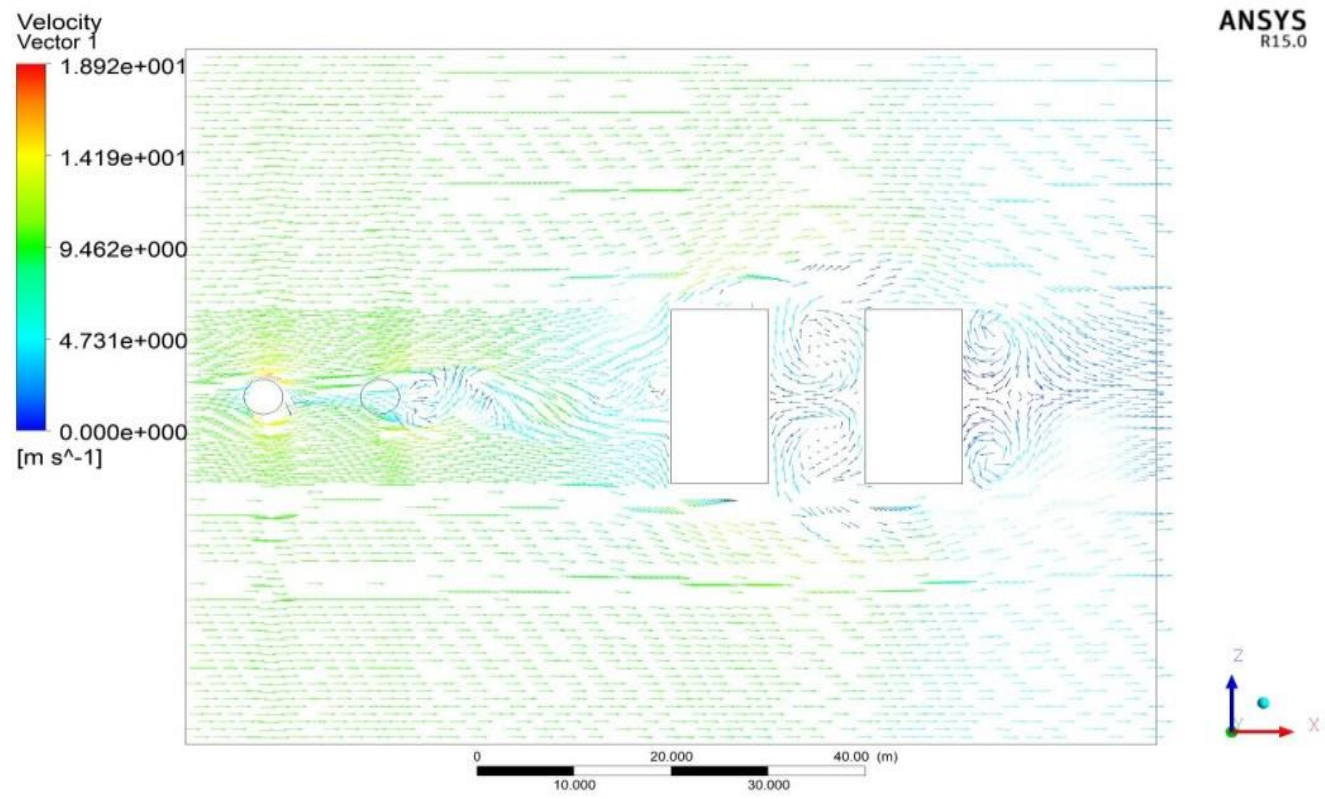

(b) 


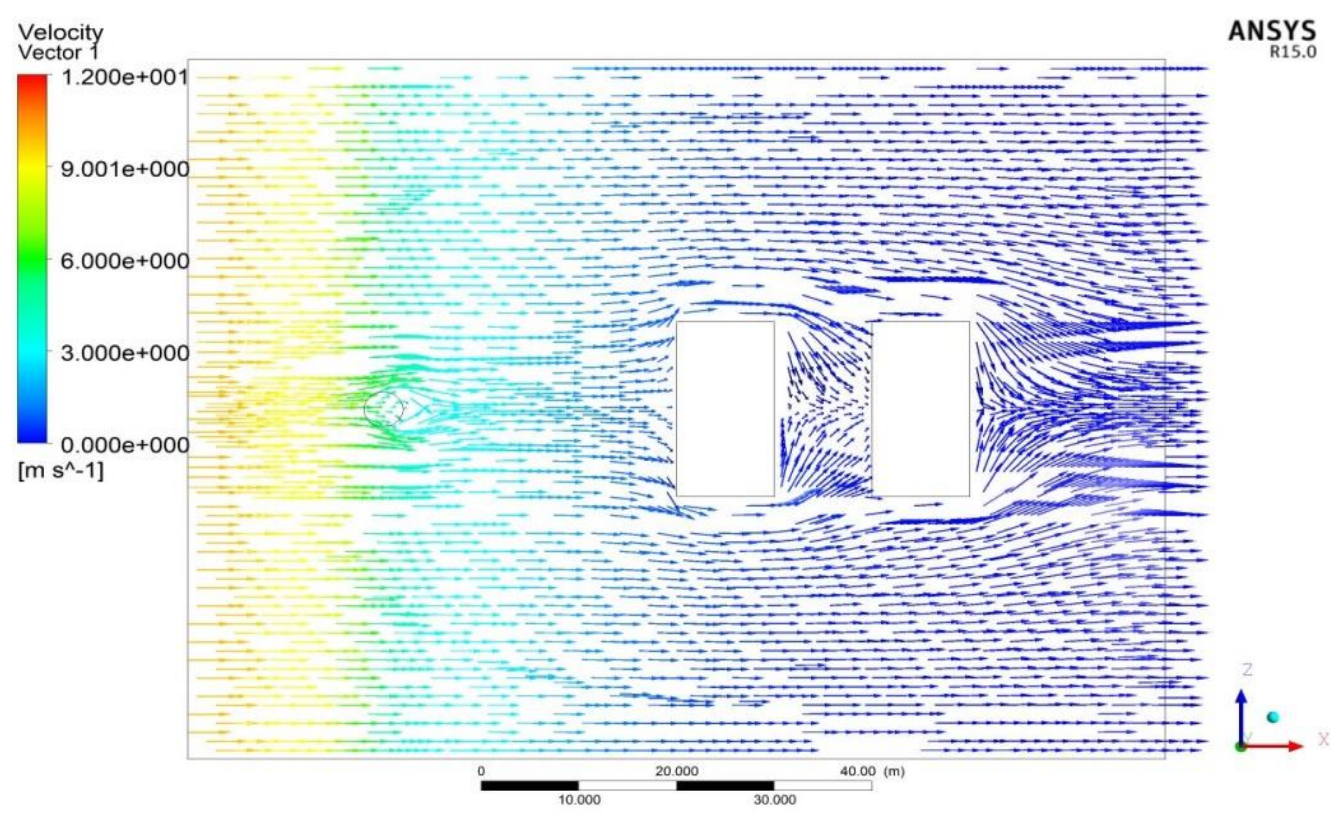

(c)

Fig.5 dispersion of the velocity vector by LES model without obstruction

(a) k- $\varepsilon$ chimney upstream, (b) LES with chimney upstream and (c) LES without chimney upstream.

With LES model of turbulence and $\mathrm{R}=0.5$ a comparison of the temperature in different altitudes have done. The hot gas move upwards by the chimney effect even with the inclination of the plume due to the wind, the temperature increases with altitude and the vortex disorder this increase in various locations (figure 6). The stratified zone whatever its altitude can cool down and dilute to about $35 \mathrm{~m}$ horizontally in maximum.

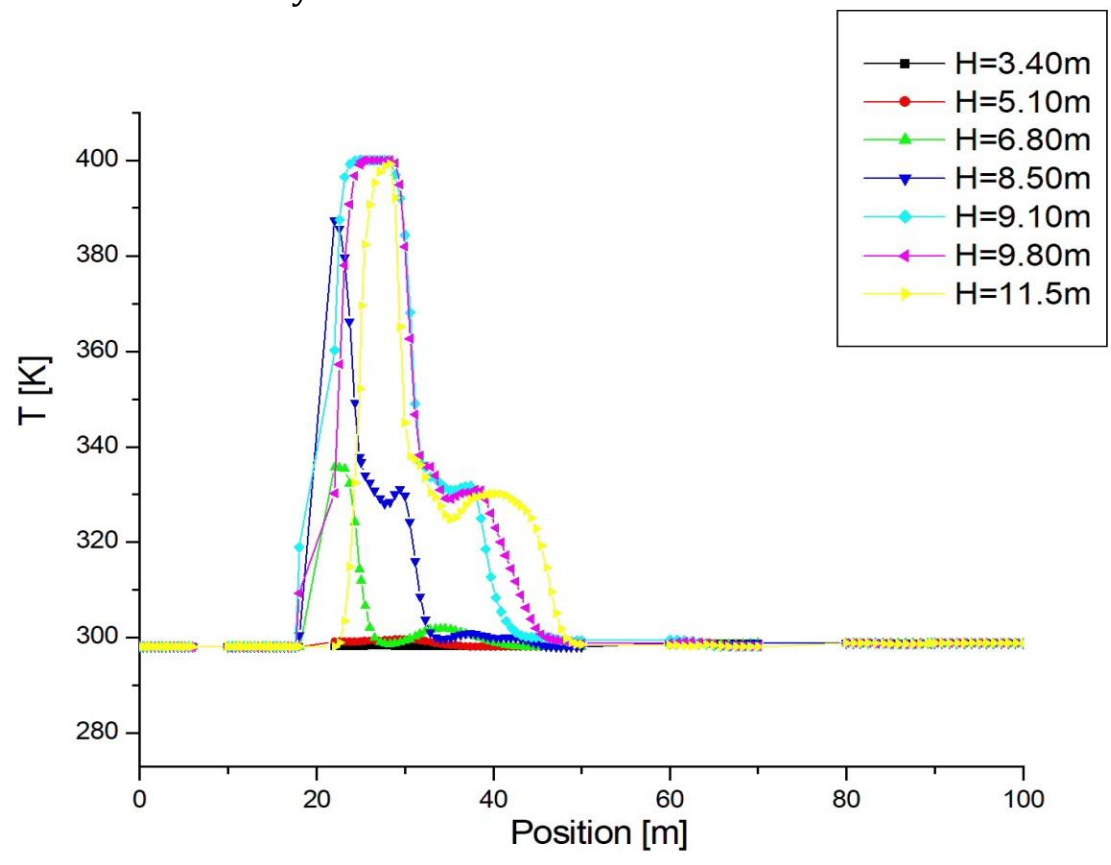

Fig. 6 Comparison of $\mathrm{CO}_{2}$ temperature for the different altitudes with LES model. 
The Reynolds stress tensor is computed using an assumption which relates the Reynolds stress tensor to the velocity gradients and the turbulent viscosity. This assumption is called the Boussinesq assumption in which we calculate turbulent viscosity often called eddy viscosity $\mu \mathrm{t}$ [Kg.ms-1]. Turbulent viscosity $\mu$ t is characteristic of the flow and not fluid, it is a sign of turbulence and too helpful to locate the turbulence vortex. The turbulent viscosity increases and reaches the maximum with the height (see figure 7.a with $\mathrm{H}=11.5 \mathrm{~m}$ ) in the vicinity of chimney $\mathrm{n}^{\circ} 1$. However, contrary and an inversely proportional evolution, that the viscosity is important in the minimal height $\mathrm{H}=8.5 \mathrm{~m}$ mostly in the vicinity of exhaust chimney.

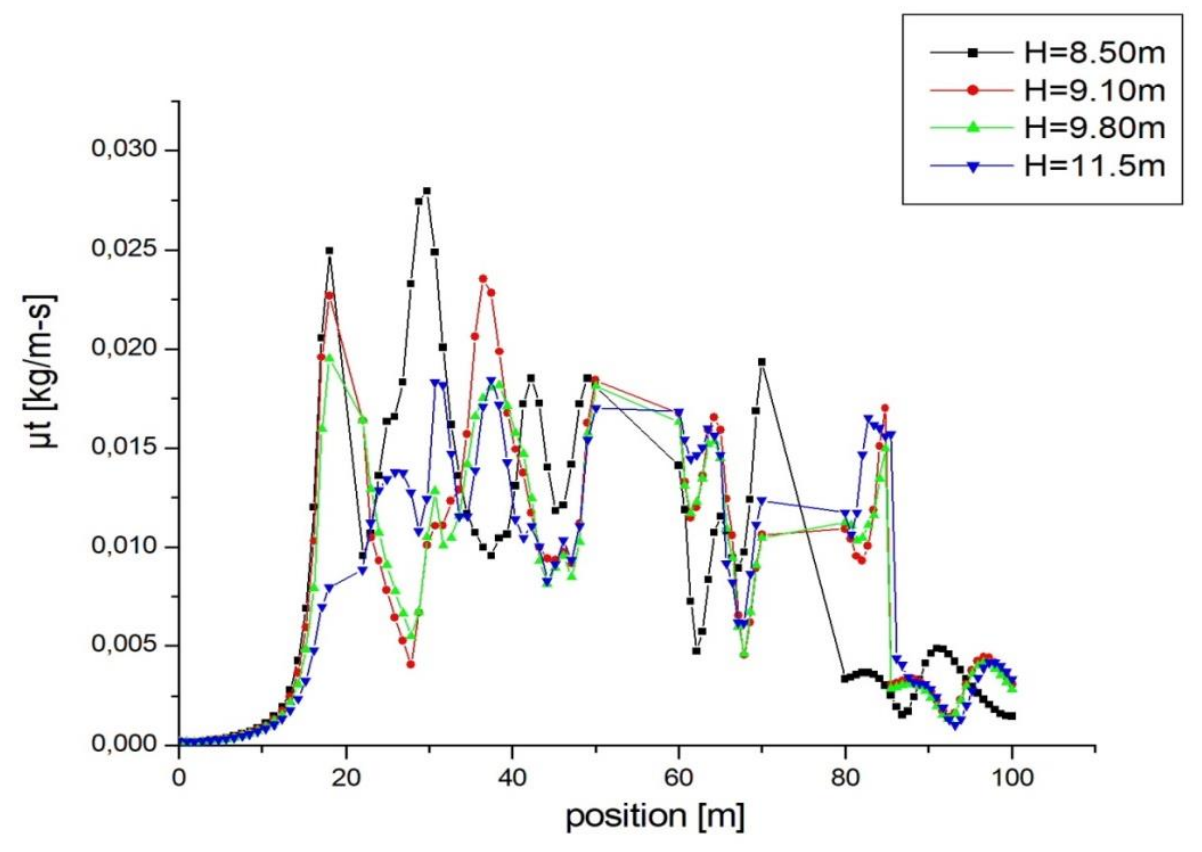

(a)

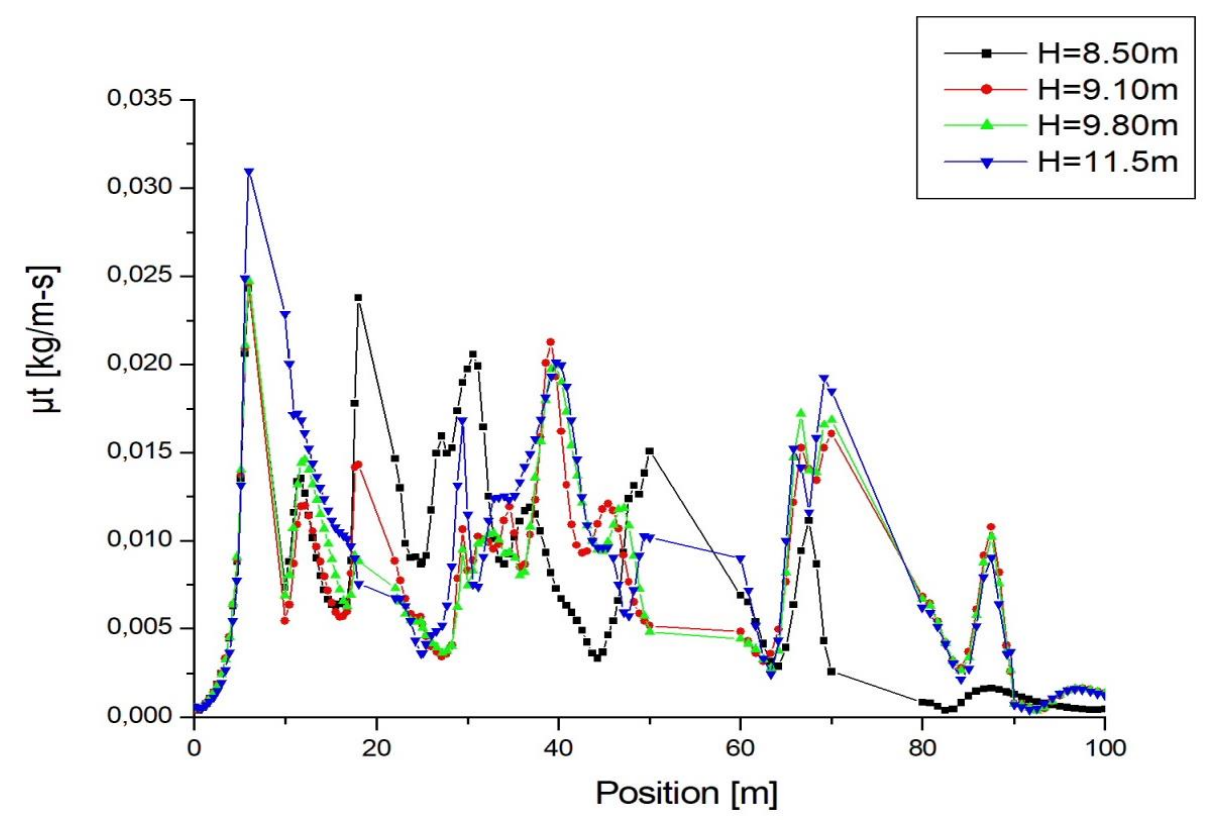

(b)

Fig. 7 the evolution of turbulent viscosity with / without chimney 1: (a) with chimney 1 (b) without it. 
Cities and industry have evolved together since the Industrial Revolution and rate of pollution and environmental degradation increase with urban grown around factories and expanding manufacturing. In last decade, many scientific researches focuses to the environmental challenges, by re-examination the interface between industrial area and cities (Berger et al., 2013; Helper et al., 2012; Leigh and Hoelzel, 2012) to understand the relationship between working and living. Wang et al. (2014) declared that flue gas discharged from the chimney with appropriate height has significant impacts on the surrounding buildings. An article focuses to U.S. cement industry, proclaim some environmental challenges and opportunities facing the cement manufacturing industry, based to the used chemistry, technology, raw materials, and energy requirements of cement manufacture were all summarized in the paper of Van Oss and Padovani (2003). A very interesting paper of Toja-Silva et al. (2018) they investigated gas dispersion, the effect of urban pattern, built density and buildings height was studied. Buildings height was found as the most influencing parameter on the gas dispersion.

\section{Conclusion}

A chimney is an architectural ventilation structure made under the role and responsibility of civil engineers. They create, improve and protect the environment presented here by an interface between an industrial zone and an urban zone through a coherent material for isolation of hot toxic exhaust gases and select a coherent height, cinematic of exhaust and placement of a chimney to optimized flue gases dispersion via stack and wind effect. Given the vast number of physical processes that must be taken into account and modeled, studying the atmospheric dispersion of a pollutant from an industrial chimney is not an easy task. We have therefore issued simplifying hypotheses for our research, such as considering the wind to have a constant direction, as we have assumed that the solid particles released, in particular, by cement plant chimneys, behave in the same way as a gas. This work consists of a numerical study of atmospheric dispersion of effluents which come from industrial stacks. We have taken as an example cement production. An investigation of pollutant dispersion was carried out by a CFD code using two turbulent models such a K- $\varepsilon$ and Large Eddy Simulation model with parametric method to test the effect of geometrical characteristics of chimney, to study the influence of the presence of an obstacle upstream or downstream of an exhaust chimney stack to study the effect of flow patterns of polluted air exhausted from chimney. We have tried to explain the different results which were obtained for several parameters such as wind conditions and manufacturing building influence and proposed a coherent method for numerical modeling such as the LES method rather than K- $\varepsilon$ based on the best parameters to identifier the flow field and their dispersion such as temperature and turbulent viscosity. Finally, a proposition of revising the shape of current cement manufacturing and preventing the construction of random manufacturing based on the optimization of atmospheric dispersion of effluent exhausted from industrial chimney and taking the imposed wind and exhaust condition

\section{Acknowledgements}

This work was prepared for initial period of PRFU project of DGRSDT, under the number [No. A11N01UN220120200010].

\section{References}

Berger, S., Sharp, P.A. and the Massachusetts Institute of Technology (MIT) Task Force on Innovation and Production (2013) A Preview of the MIT Production in the Innovation Economy Report. Cambridge, MA: Massachusetts Institute of Technology. Available at: http://web.mit.edu/pie/news/PIE_Preview.pdf.

Chen, C., Habert, G., Bouzidi, Y., \& Jullien, A. (2010). Environmental impact of cement production: detail of the different processes and cement plant variability evaluation. Journal of Cleaner Production, 18(5), 
478-485.

Gäbel, K. (2001). A life cycle process model. Simulation of Environmental, Product and Economic Performance in Cement Production.

Gäbel, K., Forsberg, P., \& Tillman, A. M. (2004). The design and building of a lifecycle-based process model for simulating environmental performance, product performance and cost in cement manufacturing. Journal of Cleaner Production, 12(1), 77-93.

Helper, S., Krueger, T., \& Wial, H. (2012). Locating American Manufacturing. Trends in the Geography of production. Metropolitan Policy Program at Brookings.

Huntzinger, D. N., \& Eatmon, T. D. (2009). A life-cycle assessment of Portland cement manufacturing: comparing the traditional process with alternative technologies. Journal of Cleaner Production, 17(7), 668-675.

Josa, A., Aguado, A., Cardim, A., \& Byars, E. (2007). Comparative analysis of the life cycle impact assessment of available cement inventories in the EU. Cement and concrete research, 37(5), 781-788.

Leigh, N. G., \& Hoelzel, N. Z. (2012). Smart growth's blind side: Sustainable cities need productive urban industrial land. Journal of the American Planning Association, 78(1), 87-103.

Malhotra, V. M. (1999). Making concrete" greener" with fly ash. Concrete international, 21(5), 61-66.

Nauclér, T., \& Enkvist, P. A. (2009). Pathways to a low-carbon economy: Version 2 of the global greenhouse gas abatement cost curve. McKinsey \& Company, 192(3).

Reijnders, L. (2007). The cement industry as a scavenger in industrial ecology and the management of hazardous substances. Journal of Industrial Ecology, 11(3), 15-25.

Summerbell, D. L., Barlow, C. Y., \& Cullen, J. M. (2016). Potential reduction of carbon emissions by performance improvement: A cement industry case study. Journal of Cleaner Production, 135, 13271339.

Toja-Silva, F., Pregel-Hoderlein, C., \& Chen, J. (2018). On the urban geometry generalization for CFD simulation of gas dispersion from chimneys: Comparison with Gaussian plume model. Journal of Wind Engineering and Industrial Aerodynamics, 177, 1-18.

Van Oss, H. G., \& Padovani, A. C. (2003). Cement manufacture and the environment part II: environmental challenges and opportunities. Journal of Industrial ecology, 7(1), 93-126.

Wang, Z. F., Xia, B., Xue, T., Liu, L. S., Wu, J. X., Wang, E. Y., \& Gou, X. (2014). Simulation Research about Impacts of Flue Gas on Surrounding Buildings. Applied Mechanics and Materials, 694, 514-519. https://doi.org/10.4028/www.scientific.net/amm.694.514

Zhiyin, Y. (2015). Large-eddy simulation: Past, present and the future. Chinese journal of Aeronautics, 28(1), 11-24. 